Research Article

\title{
New One-Dimensional Search Iteration Algorithm and Engineering Application
}

\author{
Yiping Luo $\left(\mathbb{D},{ }^{1}\right.$ Jinhao Meng $\left(\mathbb{D},{ }^{1}\right.$ Defa Wang $\left(\mathbb{D},{ }^{2}\right.$ and Guobin Xue $\mathbb{i D}^{3}$ \\ ${ }^{1}$ Institute of Water Resources and Hydro-Electric Engineering, Xi'an University of Technology, Xi'an 710048, China \\ ${ }^{2}$ Institute of Civil Engineering, Xi'an University of Technology, Xi'an 710048, China \\ ${ }^{3}$ State Grid Gansu Electric Power Company Economic Technology Research Institute, Gansu 730050, China
}

Correspondence should be addressed to Defa Wang; 854244066@qq.com

Received 7 May 2021; Revised 1 October 2021; Accepted 4 October 2021; Published 2 November 2021

Academic Editor: Mahdi Mohammadpour

Copyright (c) 2021 Yiping Luo et al. This is an open access article distributed under the Creative Commons Attribution License, which permits unrestricted use, distribution, and reproduction in any medium, provided the original work is properly cited.

In structural optimization design, obtaining the optimal solution of the objective function is the key to optimal design, and onedimensional search is one of the important methods for function optimization. The Golden Section method is the main method of one-dimensional search, which has better convergence and stability. Based on the solution of the Golden Section method, this paper proposes an efficient one-dimensional search algorithm, which has the advantages of fast convergence and good stability. An objective function calculation formula is introduced to compare and analyse this method with the Golden Section method, Newton method, and Fibonacci method. It is concluded that when the accuracy is set to 0.1, the new algorithm needs 3 iterations to obtain the target value. The Golden Section method takes 11 iterations, and the Fibonacci method requires 11 iterations. The Newton method cannot obtain the target value. When the accuracy is set to 0.01 , the number of iterations of the new method is still the least. The optimized design of the T-section beam is introduced for engineering application research. When the accuracy is set to 0.1 , the new method needs 3 iterations to obtain the target value and the Golden Section method requires 13 iterations. When the accuracy is set to 0.01 , the new method requires 4 iterations and the Golden Section method requires 18 iterations. The new method has significant advantages in the one-dimensional search optimization problem.

\section{Introduction}

Optimization problems often appear in the fields of engineering and scientific research. Many scholars have developed different optimization methods. This is because traditional optimization methods have some inherent shortcomings, such as local optimal stagnation and search space expansion [1]. In order to solve the practical problems encountered in engineering design and life, many scholars have improved the existing methods. Abbasi et al. [2] designed an improved Harris hawks (HHO) optimization algorithm to optimize the design of tapered roller bearings (TRB). Too Jingwei used HHO optimization algorithms MEHHO1 and MEHHO2 for optimal feature selection and solved the problem of feature selection in the classification of EMG signals [3]. Salvia algae group algorithm (SSA) is a bionic optimization algorithm established in 2017 based on the chain search behaviour of Salvia foraging. Nautiyal et al. [4] proposed an improved SSA algorithm based on Gaussian, Cauchy, and Levy flight, which overcomes the tendency of slow convergence and falling into suboptimal solutions and improves the global search ability. Guo et al. [5] proposed a coastal ship route planning model based on the optimized deep $Q$ network (DQN) algorithm to realize the route planning of ships during navigation. Compared with the experiment of the traditional algorithm, it has better stability and convergence, reducing the calculation time. Deng et al. designed an improved quantum evolution algorithm (QEA) based on niche coevolution strategy and enhanced particle swarm optimization (PSO), namely, IPOQEA, to solve the problem of airport gate allocation. A method of boarding gate allocation based on IPOQEA is proposed, and the effectiveness of the proposed method is verified by the actual operation data of Baiyun Airport [6]. In 
addition, Jin et al. used mathematical models to optimize the design of financial market models and circuit control systems [7-10]. Seeking the lowest cost design under the premise of meeting and application is to optimize the design. The design principle is the optimal design, the design method is the computer, and the design method is the optimal mathematical method [11]. Structural optimization has been adopted in many structural engineering practices, such as quilted structures, frame structures, bridges, hydraulic structures, and other optimization designs that have received good results. Many scholars have conducted a lot of research on the structural design of reinforced concrete beams. Liu [12] used the method of structural optimization to determine the section height of reinforced concrete beams and changed the traditional iterative calculation method of determining the beam height,so that the beam height was optimized to simplify the design and reduce the project cost. Lao [13] considered the load-bearing capacity requirements and reinforcement structure of the reinforced concrete beam section to optimize the design of the beam section and discussed the application of the 0.618 method in the optimization design. Yu and Li [14] used the improved constrained nonlinear mixed discrete variable optimization design method (MDOD) to optimize the design of reinforced concrete single-reinforced beams, taking the cost of beam unit length as the objective function and the strength of positive and oblique sections and the structural requirements of single-reinforced beams as the constraint conditions.

One-dimensional search, also known as linear search, refers to the optimization of a single-variable function and is one of the basic methods for solving unconstrained nonlinear programming problems. One-dimensional search can be used independently to solve single-variable optimization problems, and at the same time, it is a common method to solve multivariable optimization problems. Although onedimensional search itself can be used to solve many onedimensional optimization problems in science and practice, it is more used as a means of accelerating algorithm convergence and is used in conjunction with multidimensional optimization methods such as those suitable for solving large-scale unconstrained optimization. The conjugate gradient method of the problem is combined with various quasi-Newton methods to solve numerous multidimensional optimization problems. As one of the one-dimensional search methods, the Golden Section method has high accuracy and does not require high requirements for the differentiability and convergence of the function. The Golden Section method has a wide range of applicability. Zhang [15] built a high-precision correlated colour temperature calculation method based on a small-scale lookup table based on the Golden Section method, and the results showed that the algorithm has excellent performance. Kheldoun et al. [16] proposed a new method for tracking the maximum power point of photovoltaic systems based on the Golden Section method optimization technology, which ensures that the photovoltaic system can quickly converge to the maximum power point, thereby reducing energy waste. Zoubiri et al. [17] used the Golden Section method to optimize the chemical pretreatment of agroindustrial waste used to extract sugar, and the results showed that the Golden Section algorithm is a useful step to ensure the extraction of total sugar. Gao et al. [18] studied the free vibration characteristics of sandwich piezoelectric beams under elastic boundary conditions and thermal environments and introduced a search algorithm based on the Golden Section method search to calculate the required frequency parameters. Finally, the correctness and universality of the algorithm were verified by simulation software. Shi et al. [19] applied the Golden Section one-dimensional search method to unconstrained multivariate optimization problem solving, compared it with Newton's method and damped Newton's method, and proved that the Golden Section algorithm is effective and practical. Liu et al. [20] used the "0.618 method," the Fibonacci method, and the parabola method to search for the extreme points of the pendulum compound pendulum period. Through experiments, it is found that the " 0.618 method" used 15 times to measure and the Fibonacci method used 17 times to measure. The Fibonacci method has higher search accuracy than the "Golden Section method," but it does not have an advantage of search speed in comparison. However, some scholars believe that the Golden Section method had shortcomings. Qian [21] pointed out that using the Golden Section method to optimize can only be a unimodal function in the interval and the scope of application is more limited. Zhang and Chen [22] believe that the Golden Section method is represented by the division method. Although this type of method has global convergence, it does not use the properties of the function. For some functions with better analytical properties, the convergence speed of this method is too slow. Zhang et al. [23] proposed an improved one-dimensional search index optimization algorithm. The example verification results show that the index optimization method is faster than the Golden Section method. At the same time, the interval accuracy of the optimal solution is more accurate, and when the solution intervals are similar, the convergence speed is still faster than that of the Golden Section method. Since the Golden Section method shortens the length of the search interval by $61.8 \%$ each time, Liu et al. [24] believe that, from the programming perspective, the Golden Section method only needs to insert one point at a time, but the interval shortening rate is fixed at $61.8 \%$. Therefore, a midpoint method was proposed to increase the shortening rate to $51 \%$. This paper proposes a new onedimensional search algorithm based on the iterative algorithm of the Golden Section method (Algorithm 1).

\section{Background Information}

The Golden Section method is one of the main methods for accurate one-dimensional search. By taking test points and comparing function values, the search interval containing the minimum points is continuously shortened. When the interval length is shortened to a certain extent, the points on the interval all are close to the minimum, so each point on the interval can be regarded as an approximation of the minimum point. 
Given the initial search interval $\left[a_{k}, b_{k}\right]$ and accuracy requirements $\varepsilon>0$

(1) Let $\mu_{k}=a_{k}+0.618\left(b_{k}-a_{k}\right)$ and $\psi_{2}=\psi\left(\mu_{k}\right)$

(2) Let $\lambda_{k}=a_{k}+0.382\left(b_{k}-a_{k}\right)$ and $\psi_{1}=\psi\left(\lambda_{k}\right)$

(3) If $\left|b_{k}-a_{k}\right| \leq \varepsilon$, then let $x^{*}=\left(a_{k}+b_{k}\right) / 2$, otherwise transfer to (4)

(4) If $\psi\left(\lambda_{k}\right)<\psi\left(\mu_{k}\right)$, then let $b_{k}=\mu_{k}, \mu_{k}=\lambda_{k}$, and $\psi_{2}=\psi_{1}$, go to (2) If $\psi\left(\lambda_{k}\right)=\psi\left(\mu_{k}\right), a_{k}=\lambda_{k}$ and $b_{k}=\psi\left(\mu_{k}\right)$,go to (1)

If $\psi\left(\lambda_{k}\right)=\psi\left(\mu_{k}\right)$, then let $a_{k}=\lambda_{k}, \lambda_{k}=\mu_{k}$, and $\psi_{1}=\psi_{2}$, go to (5)

(5) Let $\mu_{k}=a_{k}+0.618\left(b_{k}-a_{k}\right),\left[a_{k}, b_{k}\right]$, go to (3)

Algorithm 1: The Golden Section iterative algorithm.

Theorem 1. Let $\psi$ is a unimodal function on the interval of $\left[a_{k}, b_{k}\right], \lambda_{k}, \mu_{k} \in\left[a_{k}, b_{k}\right]$; if $f\left(\lambda_{k}\right)>f\left(\mu_{k}\right)$, then for each $x \in\left[a_{k}, \lambda_{k}\right]$, there is $\psi(x) \geq \psi\left(\mu_{k}\right)$; if $f\left(\lambda_{k}\right) \leq f\left(\mu_{k}\right)$, then for each $x \in\left[\mu_{k}, b_{k}\right]$, there is $\psi(x) \geq \psi\left(\mu_{k}\right)$.

Also, the shortening rate of each iteration interval is set to 0618, so the insertion points $\lambda_{k}$ and $\mu_{k}$ correspond to

$$
\begin{aligned}
& \lambda_{k}=a_{k}+0.382\left(b_{k}-a_{k}\right), \\
& \mu_{k}=a_{k}+0.618\left(b_{k}-a_{k}\right) .
\end{aligned}
$$

Given the accuracy $\varepsilon>0$, when the interval length of a certain step $\left|b_{k}-a_{k}\right| \leq \varepsilon$ is reached, the iteration is stopped. Let $x^{*}$ be the abscissa of the extreme point in the interval, and any point within $\left[a_{k}, b_{k}\right]$ can be taken as the approximate value of the abscissa of the extreme point.

\section{New One-Dimensional Search Algorithm}

Based on the Golden Section iterative algorithm, this paper proposes a new one-dimensional search algorithm. The principle is as follows.

Suppose $f(x)$ is a continuous unimodal function in the interval of $\left[a_{k}, b_{k}\right]$ and $x^{*}$ is the abscissa of minimum point. If the abscissa of the endpoint of the interval with the smaller function value is $a_{k}$, name the endpoint $a_{k}$ and draw a line parallel to the $x$-axis through point $a_{k}$ of $f(x)$; this parallel line has an intersection with the function curve on the other side of the point $x^{*}$. Set the abscissa of this intersection as $m$, and call this point $m$. Then, draw the intersection function curve of the midperpendicular line connecting point $a_{k}$ and point $m$ at one point, set the abscissa of this intersection point as $m_{2}$, and call this point $m_{2}$. Repeat the above operation, and draw a line parallel to the $x$-axis through point $m_{2}$ of $f(x)$; this parallel line has an intersection with the function curve on the other side of the point $x^{*}$. Set the abscissa of this intersection as $m_{3}$, and call this point $m_{3}$. It can be seen that the abscissa of $x^{*}$ still falls in the interval $\left[m_{2}, m_{3}\right]$, as shown in Figure 1. Compare the absolute value of $\left|m_{2}-m_{3}\right|$ with the precision requirement $\delta$. If $\left|m_{2}-m_{3}\right| \leq \delta$, output $\left(m_{2}-m_{3}\right) / 2$ as the abscissa of the minimum point $x^{*}$. Otherwise, judge the value of $m_{2}$ and $m_{3}$.

When $m_{2}<m_{3}$, select the iterative insertion points $\xi_{k}$ and $\eta_{k}$ and let

$$
\begin{aligned}
& \xi_{k}=m_{2}+0.382\left(m_{3}-m_{2}\right), \\
& \eta_{k}=m_{2}+0.618\left(m_{3}-m_{2}\right) .
\end{aligned}
$$

Calculate the values of functions $f\left(\xi_{k}\right)$ and $f\left(\eta_{k}\right)$. If $f\left(\xi_{k}\right)>f\left(\eta_{k}\right)$, then

$$
\begin{aligned}
& a_{k+1}=\xi_{k}, \\
& b_{k+1}=m_{3}, \\
& \xi_{k+1}=\eta_{k}, \\
& \eta_{k+1}=a_{k+1}+0.618\left(b_{k+1}-a_{k+1}\right) .
\end{aligned}
$$

Calculate the values of functions $f\left(\xi_{k+1}\right)$ and $f\left(\eta_{k+1}\right)$. Get a new search interval, and $x^{*}$ still falls within the new interval $\left(\left[a_{k+1}, b_{k+1}\right], a_{k+1}=\xi_{k}, b_{k+1}=m_{3}\right)$.

If $f\left(\xi_{k}\right)<f\left(\eta_{k}\right)$, then

$$
\begin{aligned}
& a_{k+1}=m_{2}, \\
& b_{k+1}=\eta_{k}, \\
& \eta_{k+1}=\xi_{k}, \\
& \xi_{k+1}=a_{k+1}+0.382\left(b_{k+1}-a_{k+1}\right) .
\end{aligned}
$$

Calculate the values of functions $f\left(\xi_{k+1}\right)$ and $f\left(\eta_{k+1}\right)$. Get a new search interval, and $x^{*}$ still falls within the new interval $\left(\left[a_{k+1}, b_{k+1}\right], a_{k+1}=m_{2}, b_{k+1}=\eta_{k}\right)$.

When $m_{3}<m_{2}$, exchange the values of $m_{2}$ and $m_{3}$.

Repeat the above process. The length of the search interval will continue to shrink and approach zero, and finally it will converge to the minimum point $x^{*}$ steadily.

The algorithm steps of the new one-dimensional search algorithm are as follows:

The first step: given the initial search interval $\left[a_{k}, b_{k}\right]$, accuracy $\delta$, and the accuracy requirement $\delta>0$, calculate the function values $f\left(a_{k}\right)$ and $f\left(b_{k}\right)$.

The second step: if the length of the search interval is less than the accuracy, that is, $\left|b_{k}-a_{k}\right|<\delta$, then output $x^{*}=\left(a_{k}+b_{k}\right) / 2$; otherwise, go to the third step.

The third step:

When the function value $f\left(a_{k}\right)$ is less than $f\left(b_{k}\right)$, let the abscissas of point $m, m_{2}$, and $m_{3}$ be 


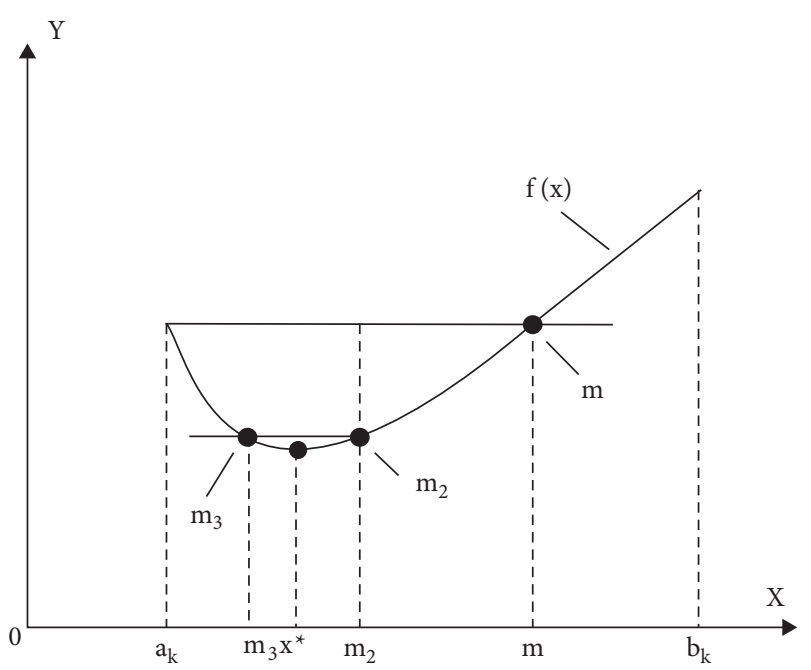

Figure 1: Schematic diagram of the new one-dimensional search algorithm.

$$
\begin{gathered}
m=a_{k}-\frac{2 f^{\prime}\left(a_{k}\right)}{f^{\prime \prime}\left(a_{k}\right)}, \\
m_{2}=a_{k}-\frac{f^{\prime}\left(a_{k}\right)}{f^{\prime \prime}\left(a_{k}\right)}, \\
m_{3}=m_{2}-\frac{2 f^{\prime}\left(m_{2}\right)}{f^{\prime \prime}\left(m_{2}\right)} .
\end{gathered}
$$

Judging:

When the distance between $m_{2}$ and $m_{3}$ is less than the accuracy $\quad\left(\left|m_{3}-m_{2}\right| \leq \delta\right)$, then output $x^{*}=\left(m_{2}+m_{3}\right) / 2$.

When $m_{3}>m_{2}$, let the abscissa of the iterative insertion point be as equation (2); otherwise (when $m_{3}<m_{2}$ ), exchange the values of $m_{2}$ and $m_{3}$ and let the iterative insertion point's abscissa be equal to equation (2).

Calculate the values of functions $f\left(\xi_{k}\right)$ and $f\left(\eta_{k}\right)$, go to the fourth step.

The fourth step:

When $f\left(\xi_{k}\right)>f\left(\eta_{k}\right)$, let the iterative insertion point's abscissa be equal to equation (3).

Calculate the values of functions $f\left(\xi_{k+1}\right)$ and $f\left(\eta_{k+1}\right)$. Otherwise, when $f\left(\xi_{k}\right) \leq f\left(\eta_{k}\right)$, let the iterative insertion point's abscissa be equal to equation (4).

Calculate the values of functions $f\left(\xi_{k+1}\right)$ and $f\left(\eta_{k+1}\right)$. Let $k=k+1$, go to the second step.

The fifth step:

When $f\left(a_{k}\right)>f\left(b_{k}\right)$, let the abscissas of point $m, m_{2}$, and $m_{3}$ be

$$
\begin{aligned}
& m=b_{k}-\frac{2 f^{\prime}\left(b_{k}\right)}{f^{\prime \prime}\left(b_{k}\right)}, \\
& m_{2}=a_{k}-\frac{f^{\prime}\left(b_{k}\right)}{f^{\prime \prime}\left(b_{k}\right)}, \\
& m_{3}=m_{2}-\frac{2 f^{\prime}\left(m_{2}\right)}{f^{\prime \prime}\left(m_{2}\right)} .
\end{aligned}
$$

When the distance between $m_{2}$ and $m_{3}$ is less than the accuracy $\quad\left(\left|m_{3}-m_{2}\right| \leq \delta\right)$, then output $x^{*}=\left(m_{2}+m_{3}\right) / 2$.

When $m_{3}>m_{2}$, let the abscissa of the iterative insertion point be as equation (2); otherwise (when $m_{3}<m_{2}$ ), exchange the values of $m_{2}$ and $m_{3}$, and let the iterative insertion point's abscissa be equal to equation (2).

Calculate the values of functions $f\left(\xi_{k}\right)$ and $f\left(\eta_{k}\right)$, go to the fourth step.

\section{Verification Analysis}

For testing the calculation effect of the new one-dimensional search algorithm, a calculation example $f(x)=x^{3}-11 x^{2}+$ $4 x+60$ is used to compare the calculation accuracy and iterative speed. Common one-dimensional search methods include Fibonacci method and Newton method. Using the new one-dimensional search method and other three kinds of methods to search the minimum points of the function, the calculation results of various methods are shown in Table 1 .

Figure 2 shows that the calculated value of the Golden Section method iteration process fluctuates around the exact value in the early stage. As the iterative process proceeds, the calculated value finally converges to the minimum value and basically coincides with the exact value, while the iterative process is generally stable. However, the number of iterations is the largest and the convergence speed is the slowest among the new algorithm, Fibonacci method, and Newton method. Besides, Golden Section method's relative error decreases less significantly as the accuracy requirements increase. The Fibonacci method also has large fluctuations in the early iteration process. After nine iterations, the calculation results converge to a minimum value and the iterative calculation process is stable. In addition, the number of iterations of the Fibonacci method is roughly equivalent to the Golden Section method, but the initial convergence speed is slower than the Golden Section method. The relative error did not change significantly with the increase in accuracy requirements, but it is still better than the Golden Section method. When the position of the initial point of Newton method is far from the minimum point in the interval, its convergence rate may be slow, even not converge, or may converge to a nonlocal minimum point. When 
TABle 1: Comparison of the new algorithm with the Golden Section method, Fibonacci method, and Newton method.

\begin{tabular}{|c|c|c|c|}
\hline \multicolumn{2}{|c|}{ Precision } & 0.1 & 0.01 \\
\hline \multicolumn{2}{|c|}{ First try interval } & {$[1,10]$} & {$[4,10]$} \\
\hline \multirow{3}{*}{ New search algorithm } & Number of iterations & 3 & 6 \\
\hline & Output result & 7.144895841400396 & 7.146757361917681 \\
\hline & Relative error & 0.000262076 & 0.000001606 \\
\hline \multirow{3}{*}{ Fibonacci method } & Number of iterations & 11 & 14 \\
\hline & Output result & 7.14164624183007 & 7.14893407443889 \\
\hline & Relative error & 0.000716771 & 0.000302967 \\
\hline \multirow{3}{*}{ Golden Section method } & Number of iterations & 11 & 15 \\
\hline & Output result & 7.145507046801479 & 7.147822394074800 \\
\hline & Relative error & 0.000176554 & 0.000147417 \\
\hline \multirow{3}{*}{ Newton method } & Number of iterations & 3 & 7 \\
\hline & Output result & 0.186563842345446 & 7.146768836448334 \\
\hline & Relative error & 0.973895358 & $2.02637 \mathrm{E}-11$ \\
\hline
\end{tabular}

*True value is 7146768836303518 (15 decimal places are reserved).

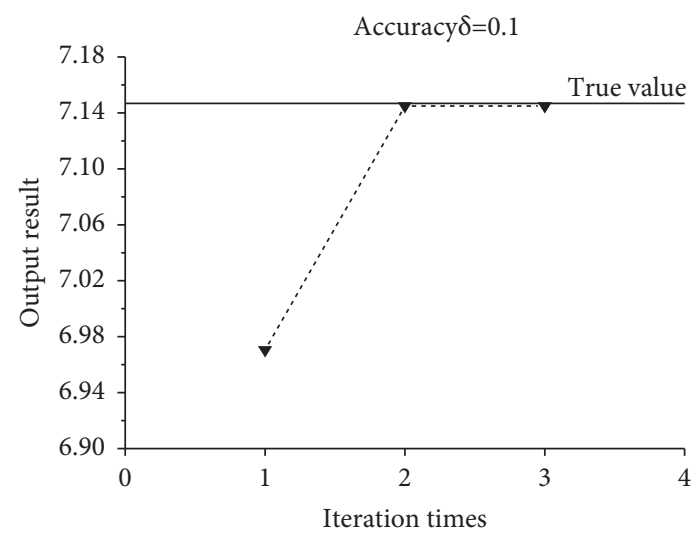

(a)

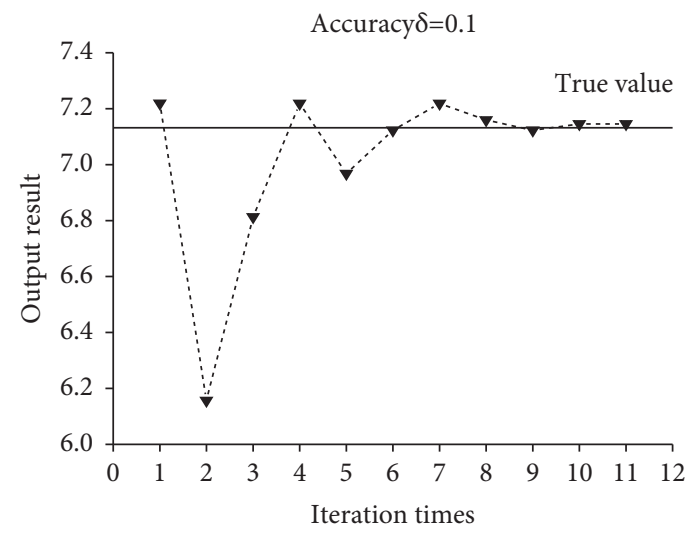

(c)

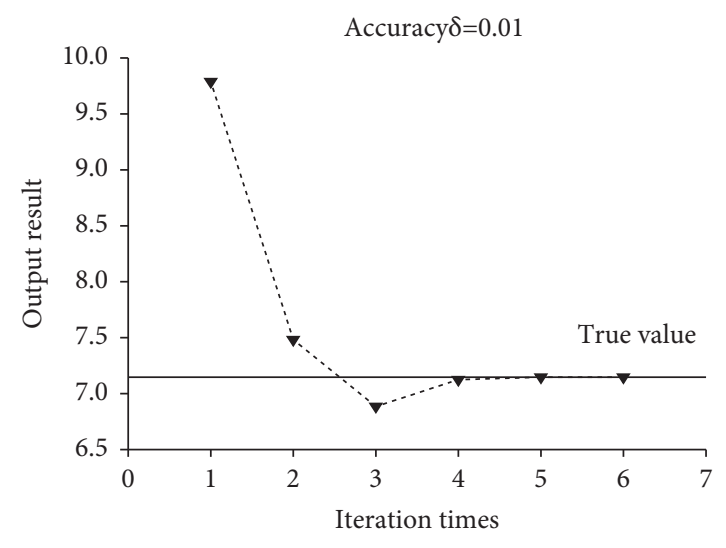

(b)

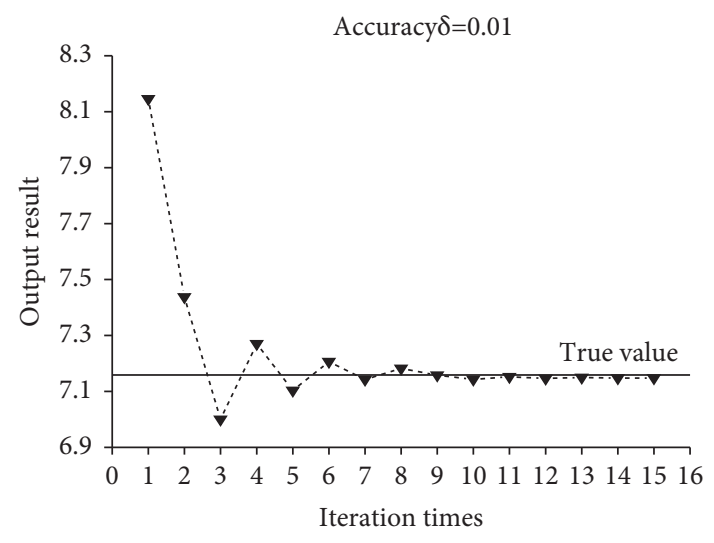

(d)

FIgURE 2: Continued. 


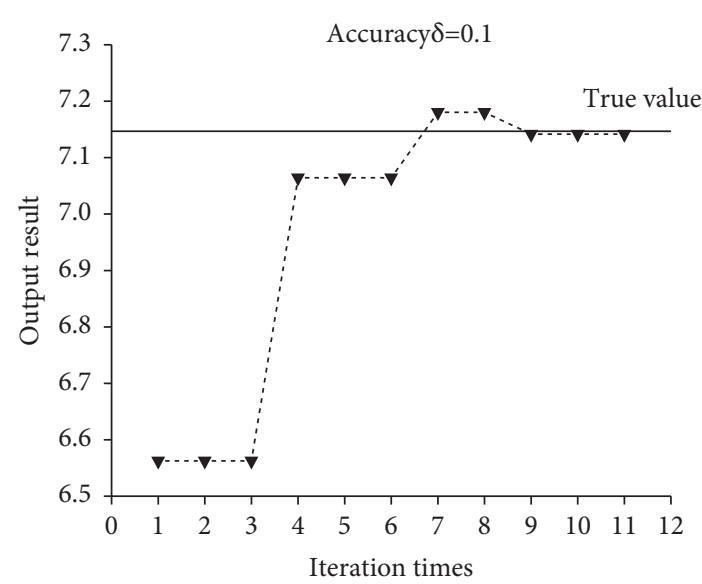

(e)

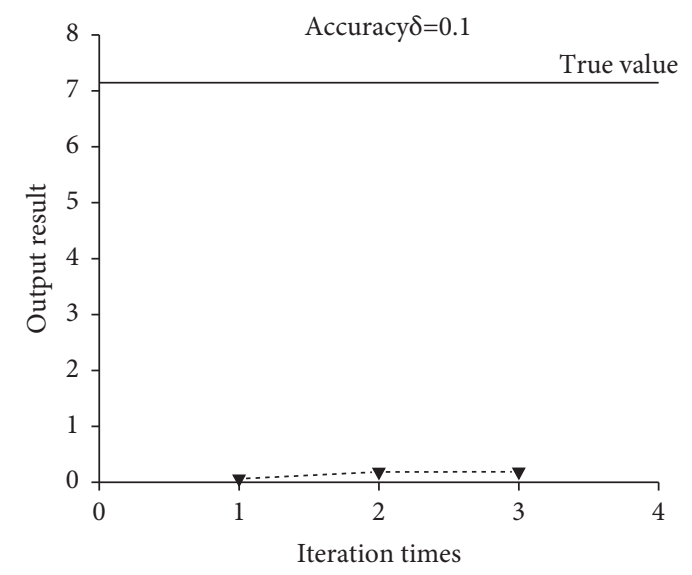

$(\mathrm{g})$

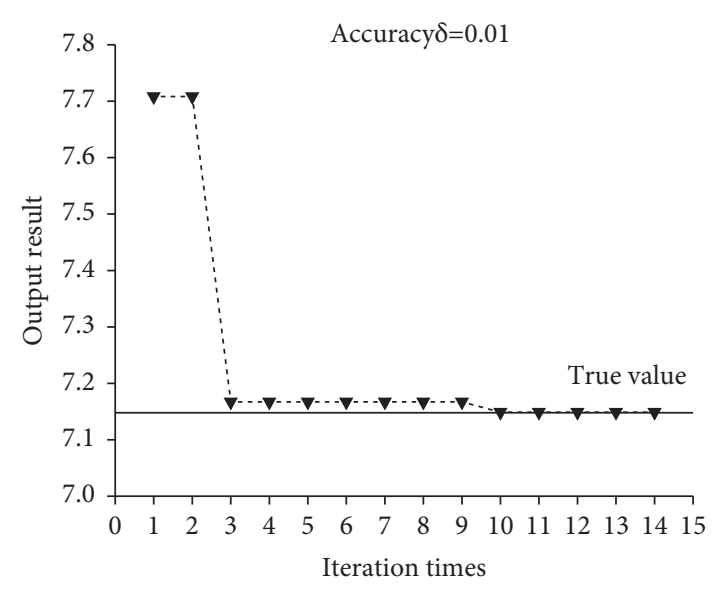

(f)

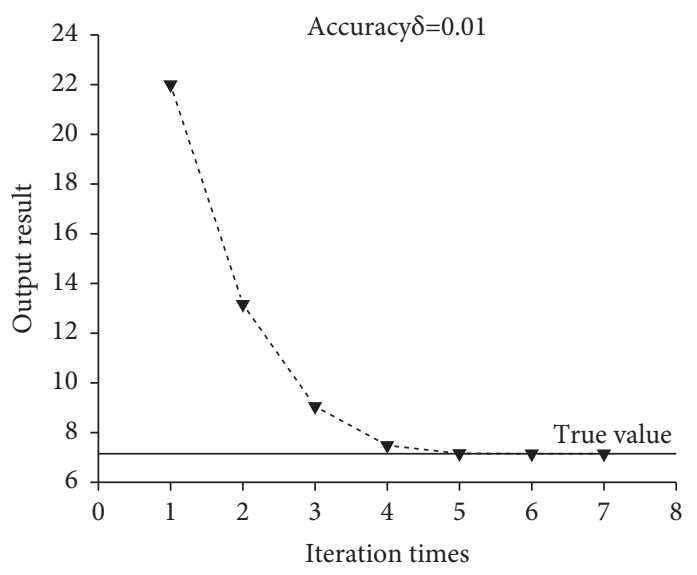

(h)

Figure 2: The relationship between the number of iterations of various algorithms and the true value. (a) New algorithm iteration results at an accuracy of 0.1. (b) New algorithm iteration results at an accuracy of 0.01. (c) Golden Section method results at an accuracy of 0.1. (d) Golden Section method results at an accuracy of 0.01. (e) Fibonacci method results at an accuracy of 0.1. (f) Fibonacci method results at an accuracy of 0.01 . (g) Newton method results at an accuracy of 0.1. (h) Newton method results at an accuracy of 0.01 .

the initial point in the interval is 1 , the calculated result is far from the true value and the stability is poor. However, when the interval is selected properly, its convergence is good and the stability is highly accurate at 0.01 . After four iterations, the calculated value converges to the exact value. The iteration rate is increased by more than $50 \%$ compared to both the Golden Section method and Fibonacci method. The relative error is the smallest of the four methods, mainly because the Newton method has a local second-order convergence speed. The new one-dimensional search iterative algorithm converges to a minimum value after three iterations. Compared with the Golden Section method and the Fibonacci method, the new iterative algorithm improved the iteration time by $75 \%$. It also has the advantage of fast convergence speed and stable convergence when compared with Newton method, and it can maintain a faster convergence speed, even under different accuracy requirements. Furthermore, as accuracy requirements increase, the absolute error decreases significantly.

In general, when the accuracy is set to 0.1 , the new algorithm requires three iterations to obtain the target value, the Golden Section method requires 11 iterations, and the Fibonacci method also requires 11 iterations. The Newton method cannot obtain the target value. When the accuracy is set to $0.01,6$ iterations are needed for the new algorithm to obtain the target value, 15 iterations for the Golden Section method, 14 iterations for the Fibonacci method, and 7 iterations for the Newton method. There is no doubt that the new one-dimensional search iterative algorithm has the advantages of fast iteration speed, good convergence, and high stability of calculation results.

\section{Case Analysis}

As shown in Figure 3, T-section beam (also called T-beam or T-shaped beam) is widely used in engineering structures, for example, T-beams formed by cast-in-place rib beams and floor slabs and independent T-beams in prefabricated components. Some other prefabricated beams in the form of cross sections, such as I-beam crane beams and thin-belt roof beams, are also considered according to the T-section beam members. Compared with the rectangular section 


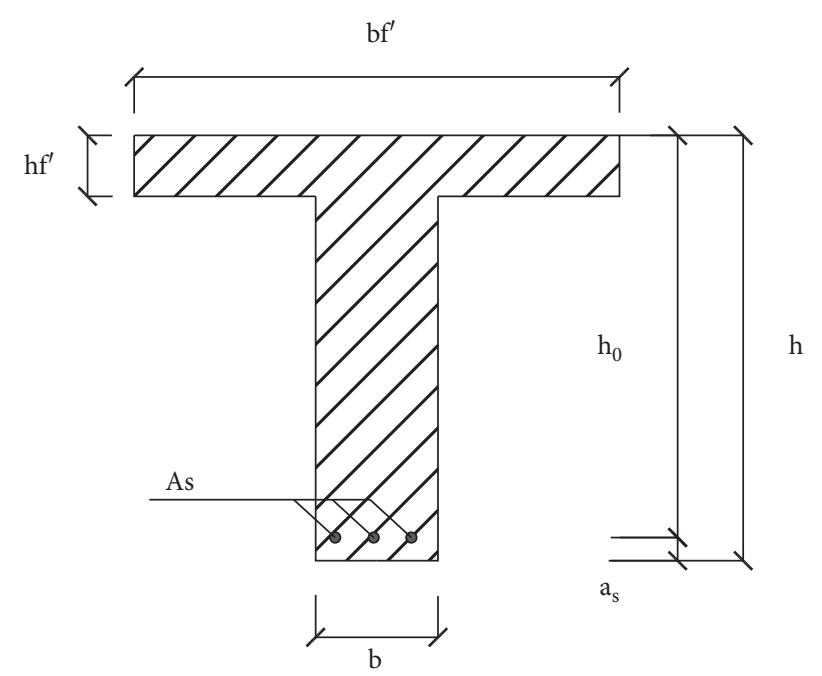

FIgURE 3: T-shaped section in engineering structure.

beam, the T-section beam's ultimate bearing capacity is not affected, and it saves concrete materials, reduces its own weight, and has certain economic benefits. This example is based on the relevant literature and Chinese national code GB50010-2010 "Specifications for the Design of Concrete Structure," taking the lowest cost as the goal and factoring in the strength and structural requirements; the section optimization design of the $\mathrm{T}$-shaped reinforced concrete beam is the preliminary design for reference.

5.1. Establishment of Mathematical Model. Assume that the span of a simply supported beam is $l$, the maximum bending moment of the interface under load is $M_{\max }$, and the maximum shear force is $V_{\max }$. Considering that the section meets the strength conditions and the structural requirements of the beam in the code, the optimization is to make the T-section beam with the least amount of concrete and steel, regardless of the cost of manual production.

Take the price $C$ of the beam of unit length as the objective function:

$$
C=c_{h}\left[b\left(h_{0}+a_{s}\right)+\left(b_{f}^{\prime}-b\right) h_{f}^{\prime}\right]+c_{s} A_{s},
$$

where $c_{h}$ is the unit price of concrete (refer to Xi'an concrete price), $c_{s}$ is the rebar unit price (refer to Xi'an rebar price), $h_{0}$ is the effective height of T-section beam, $h_{f}^{\prime}$ is the flange height, $A_{s}$ is the rebar cross-sectional area, $b$ is the web width, $a_{s}$ is the protective layer thickness, and $b_{f}^{\prime}$ is the flange width.

\subsection{Constraints. Flexural strength requirements:}

For type I sections,

$$
M \leq \alpha_{1} f_{c} b_{f}^{\prime} x\left(h_{0}-\frac{x}{2}\right),
$$

where $M$ is the design value of bending moment; $\alpha_{1}$ is the calculation coefficient, when the concrete not exceeding C50, 10 is used and when it is C80, take the value as 0.94 , and the middle-grade concrete is determined by linear interpolation; $f_{c}$ is the design value of concrete axial compressive strength; and $x$ is the height of the interface compression zone.

For type II sections,

$$
M \leq \alpha_{1} f_{c}\left(b_{f}^{\prime}-b\right) h_{f}^{\prime}\left(h_{0}-\frac{h_{f}^{\prime}}{2}\right)+\alpha_{1} f_{c} b x\left(h_{0}-\frac{x}{2}\right) .
$$

The meaning of each coefficient in the formula is the same as formula (8).

Shear strength requirements:

For T-section or I-section simply supported beams,

$$
V \leq 0.3 \beta_{c} f_{c} b h_{0},
$$

where $\beta_{c}$ is concrete strength influence coefficient: when the concrete strength grade does not exceed C50, it is taken as 1.0, and when the concrete strength grade is $\mathrm{C} 80$, it is taken as 0.8 ; and the middle-grade concrete is determined by linear interpolation.

5.3. Reinforcement Limitation Requirements. Reinforcement ratio $M_{u}$ should meet the following equation:

$$
M_{u}=\alpha_{s b} \alpha_{1} f_{c} b h_{0}^{2} \approx 0.4 \alpha_{1} f_{c} b h_{0}^{2},
$$

where $\alpha_{s b}$ is the maximum resistance to bending moment coefficient of the section. The meaning of the other coefficients in the formula is the same as the above formula.

Furthermore, the minimum reinforcement ratio $\rho_{\min }$ should also meet the following equation:

$$
\frac{A_{s}}{b h_{0}} \geq \rho_{\text {min }} .
$$

5.4. Simplification of the Optimization Model. Since the optimization design of reinforced concrete beams is a multivariable, multiconstrained, and nonlinear optimization problem, if starting from the actual engineering, based on 
the analysis of design and use experience, some variables will be used as predetermined parameters to reduce the number of design variables. The calculation of certain parameters in the objective function and constraint conditions is simplified to reduce the degree of nonlinearity, which makes the optimization design problem simple and easy. Therefore, this article deals with the optimization design of reinforced concrete T-section beams as follows:

(1) Beam web section width $b$ : in general engineering design, it is usually selected according to structural requirements. Therefore, this paper considers it as a predetermined parameter and not as a design variable.

(2) In order to reduce the design variables, the optimization is based on the rectangular cross section of a single reinforcement. The effect of the reinforcement in the compression zone is not considered, and only the vertical reinforcement according to the structure is considered.

(3) In order to reduce the optimization parameters, in the constraint condition of the shear capacity of the inclined section, the bending reinforcement is not considered, only the shear effect of the stirrup $\left(A_{s \mathrm{v}}\right)$ is considered, and the stirrup is not optimized as a design variable.

(4) Restrictions on crack development width, crack resistance, and deflection are not considered as constraints. As for general reinforced concrete beams, the above conditions can basically meet the requirements, so the impact on the optimization design results is not great, but it makes the optimization process simpler.

(5) In order to further simplify the optimization process and reduce design variables, the cross-sectional area of the cross-section steel bar when the aspect ratio of the web section is not greater than 2.5 is replaced by the approximate formula [25]:

$$
A_{s}=\frac{M}{\gamma_{s} f_{y} h_{0}}
$$

where $\gamma_{s}$ is the internal force arm coefficient, and it is taken as 0.9 .

After the above treatment, the optimization design problem of reinforced concrete $\mathrm{T}$-shaped beams becomes even simpler. In the objective function, this article only considers the main comparable factors that affect the cost of reinforced concrete $\mathrm{T}$-section beams; that is, only the cost of tensile steel and concrete in the beam is included. As for other factors such as stirrup, structural reinforcement, concrete formwork, and labor costs, after the structural designplan is determined, these factors have little effect on the cost of reinforcedconcrete beams and should not be counted. If it is strictly required, when considering the cost of the beam and calculating its cost, it can be reflected in the unit price of concrete and steel bar in the tensile zone, and it can be converted into the relevant unit price.

From formulas (11) and (12), the lower limit of the effective height $h_{0}$ is as follows:

$$
h_{0}=\max \left(\frac{V_{\max }}{0.3 \beta_{c} f_{c} b h_{0}}, \sqrt{\frac{M_{\max }}{0.4 \alpha_{1} f_{c} b}}\right) .
$$

From formula (12), the lower limit of reinforcement cross-sectional area $A_{s}$ is as follows:

$$
A_{s} \geq b h_{0} \rho_{\min } .
$$

Substituting formula (12) into (6), we obtain the lowest cost function:

$$
\min C=c_{h}\left[b\left(h_{0}+a_{s}\right)+\left(b_{f}^{\prime}-b\right) h_{f}^{\prime}\right]+c_{s} \frac{M_{\max }}{\gamma_{s} f_{y} h_{0}} .
$$

5.5. Instance Import. The main beam of a T-shaped beam of a monolithic rib beam floor is known as $b_{f}^{\prime}=2200 \mathrm{~mm}$, $h_{f}^{\prime}=80 \mathrm{~mm}$, and $b=300 \mathrm{~mm}$, and the environmental category is a category, $a_{s}=25 \mathrm{~mm}$, choose C30 concrete. For concrete, set the unit price of concrete at $350 \mathrm{yuan} / \mathrm{m}^{3}$, the unit price of steel bar at 31,000 yuan $/ \mathrm{m}^{3}$, and HRB335 grade steel bar $M_{\text {max }}=400 \mathrm{KN} \cdot m$ and $V_{\max }=450 \mathrm{KN} \cdot m$, and try to optimize the design of the section with the lowest cost.

Substituting the above known data into formula (16) gives

$$
\min C=0.105 h_{0}+55.825+\frac{45926}{h_{0}} .
$$

MATLAB has powerful numerical calculation functions, which can be used to solve the one-dimensional optimization problem. The objective function in the problem is solved by a new one-dimensional search iterative algorithm programming operation and compared with the Golden Section method.

The flowchart of the new algorithm is shown as Figure 4.

Table 2 shows that the calculation result of the new onedimensional search algorithm is $h_{0}=661.38 \mathrm{~mm}$ (take two significant digits), and the cost objective function $\min C=194.7$ yuan is taken into consideration. Considering that the actual project can take $h_{0}=675 \mathrm{~mm}$, the actual height of the T-section beam plus the thickness of the protective layer is $700 \mathrm{~mm}$ and the corresponding cost is 195 yuan per meter.

From the calculation results in Table 2, it is not difficult to see that the Golden Section method has a small number of iterations when the accuracy requirement is 1.0 and iteration times are 13 and stably converge to the target value eventually; when the accuracy requirement is set to 0.1 , the calculation result is closer to the true value. However, the number of iterations increased significantly to 18 . The new one-dimensional search method has three iterations when 


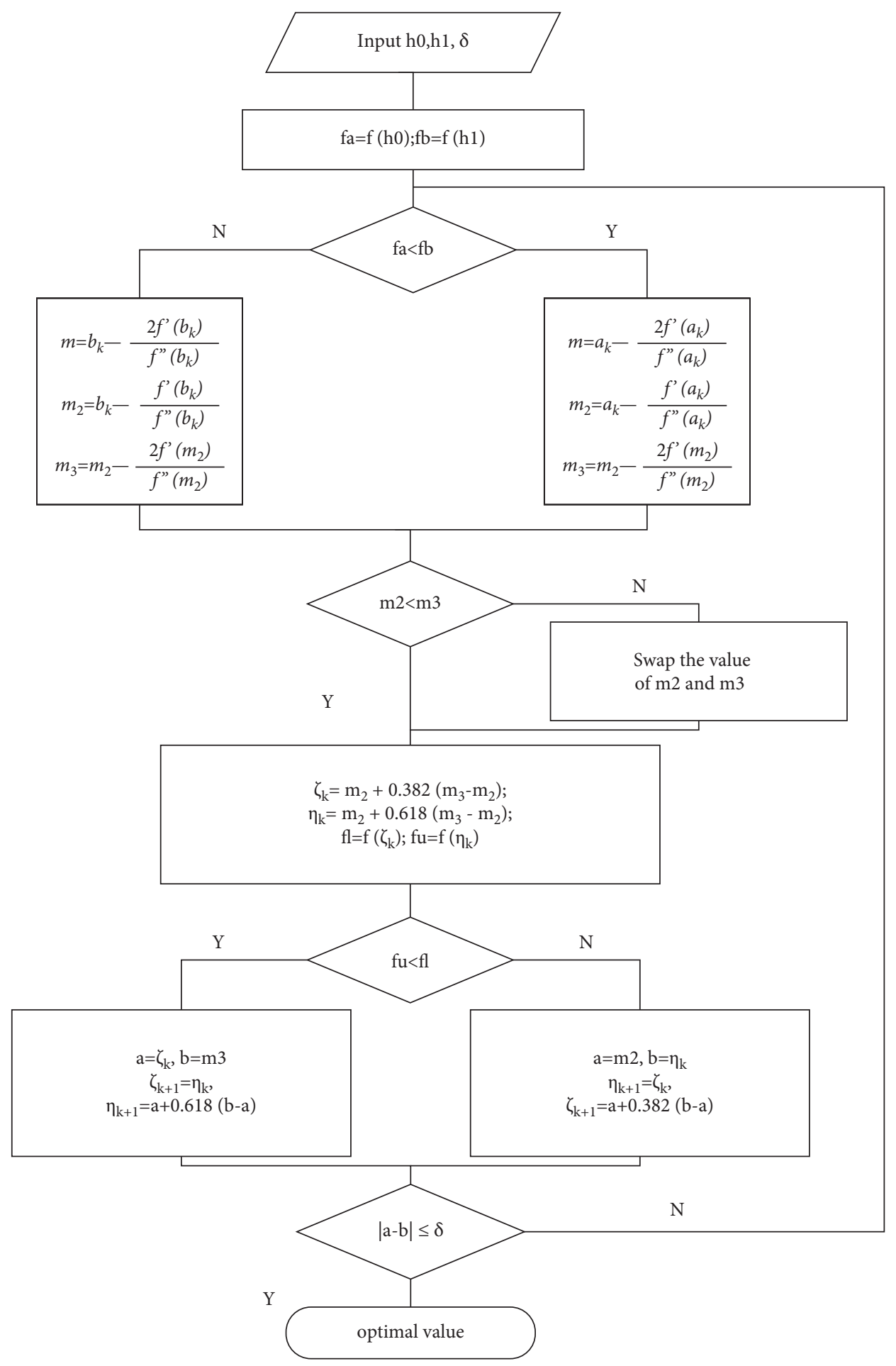

FIGURE 4: Algorithm program flowchart.

the accuracy of the calculation of the objective function is 1.0 , which is only $25 \%$ iteration time of the Golden Section method, which is much better than the latter. The accuracy of the calculation result is higher than that of the Golden Section method. It meets the actual requirements of the project; when the accuracy requirement is 1.0 , the number of iterations is 4 times, and the accuracy requirements are improved without a significant increase in the number of iterations. In general, the new one-dimensional search iterative algorithm has fast iteration speed and accurate calculation results, which greatly reduce the calculation workload. The calculation results can be used as a reference 
TABle 2: Program running results.

\begin{tabular}{|c|c|c|c|}
\hline Precision & & 1 & 0.1 \\
\hline \multirow{4}{*}{ New algorithm } & First try interval & {$[500,750]$} & {$[500,750]$} \\
\hline & Number of iterations & 3 & 4 \\
\hline & Output result & 661.3765 & 661.3765 \\
\hline & Absolute error & 0.0215 & 0.0215 \\
\hline \multirow{4}{*}{ Golden Section method } & First try interval & {$[500,750]$} & {$[500,750]$} \\
\hline & Number of iterations & 13 & 18 \\
\hline & Output result & 661.4710 & 661.3457 \\
\hline & Absolute error & 0.116 & 0.0093 \\
\hline
\end{tabular}

${ }^{*}$ The true value of the number calculation is about 6613550 (4 decimal places are reserved).

for the initial value of the optimal design of the T-beam section in actual engineering.

\section{Conclusion}

Aiming at the Golden Section method in the one-dimensional search method, this paper proposes a new one-dimensional search iterative method by analysing the principle of Golden Section method. The numerical example proves that the new method has faster iteration speed than the classic Newton method, Golden Section method, and Fibonacci method. The number of iterations is only $25 \%$ of the Golden Section method. It has better convergence, and the calculation is stable and reliable. The advantage is more obvious, and the error is smaller. Finally, an engineering example on the optimization design of the T-shaped reinforced concrete beam section was verified, and the following conclusions were reached:

(1) Under the same accuracy requirements, the new algorithm accelerates the interval convergence speed, reduces the number of iterations, and has good numerical stability

(2) When the accuracy of the extreme point of the function being calculated is not high, the calculation time can be saved and the amount of calculation can be reduced

(3) In the engineering example of the optimal design of the T-shaped concrete beam, the new one-dimensional search calculation result is more accurate, the number of iterations is relatively small, and it has certain theoretical and practical application value

\section{Data Availability}

The data used to support the findings of this study are available from the corresponding author upon request.

\section{Conflicts of Interest}

The authors declare that there are no conflicts of interest regarding the publication of this paper.

\section{Acknowledgments}

This study was funded by the Yulin City's 2020 Science and Technology Plan Project (Nos. CXY-2020-081 and CXY-
2020-080) and the National Natural Science Foundation of China Youth Fund Project (No. 51808445).

\section{References}

[1] H. Chen, M. Wang, and X. Zhao, "A multi-strategy enhanced sine cosine algorithm for global optimization and constrained practical engineering problems," Applied Mathematics and Computation, vol. 369, no. 1, Article ID 124872, 2020.

[2] A. Ahmad, F. Behnam, S. Polat, A. Asghar Heidari, H. Chen, and R. Tiwari, "Multi-strategy Gaussian Harris hawks optimization for fatigue life of tapered roller bearings," Engineering with Computers, 2021.

[3] J. Too, L. Guoxi, and C. Huiling, "Memory-based Harris hawk optimization with learning agents: a feature selection approach," Engineering with Computers, 2021.

[4] B. Nautiyal, R. Prakash, V. Vimal, L. Guoxi, and C. Huiling, "Improved Salp Swarm Algorithm with mutation schemes for solving global optimization and engineering problems," Engineering with Computers, 2021.

[5] S. Guo, X. Zhang, Y. Du, Y. Zheng, and Z. Cao, "Path planning of coastal ships based on optimized DQN reward function," Journal of Marine Science and Engineering, vol. 9, no. 2, p. 210, 2021.

[6] W. Deng, J. Xu, H. Zhao, and Y. Song, "A novel gate resource allocation method using improved PSO-based QEA," IEEE Transactions on Intelligent Transportation Systems, vol. 1-9, 2020.

[7] T. Jin, H. Ding, H. Xia, and J. Bao, "Reliability index and Asian barrier option pricing formulas of the uncertain fractional first-hitting time model with Caputo type," Chaos, Solitons \& Fractals, vol. 142, Article ID 110409, 2021.

[8] T. Jin, H. Ding, L. Bo, X. Hongxuand, and X. Chenxia, "Valuation of interest rate ceiling and floor based on the uncertain fractional differential equation in Caputo sense," Journal of Intelligent and Fuzzy Systems, vol. 40, no. 3, 2021.

[9] T. Jin, H. Xia, and H. Chen, "Optimal control problem of the uncertain second-order circuit based on first hitting criteria," Mathematical Methods in the Applied Sciences, vol. 44, 2021.

[10] T. Jin, H. Ding, H. Xia, and J. Bao, "Reliability index and Asian barrier option pricing formulas of the uncertain fractional first-hitting time model with Caputo type," Solitons \& Fractals, vol. 21, no. 9, 2020.

[11] S. Qin, "Optimization design and information construction of landslide engineering treatment," Chinese Journal of Geological Hazard and Control, vol. 2-10, 1999.

[12] Y. Liu, "Optimal design of reinforced concrete beam section," Fujian Architecture, vol. 13, pp. 38-37, 1997.

[13] C. Lao, "Optimal design of reinforced concrete beam," Nonferrous Metal Design, vol. 33-38, 1996. 
[14] M. Yu and Q. Li, "Optimal design of reinforced concrete single reinforced beams," Industrial Architecture, vol. 13, pp. $48-51,2000$.

[15] F. Zhang, "High-accuracy method for calculating correlated color temperature with a lookup table based on golden section search," Optik, vol. 193, Article ID 163018, 2019.

[16] A. Kheldoun, R. Bradai, R. Boukenoui, and A. Mellit, "A new golden section method-based maximum power point tracking algorithm for photovoltaic systems," Energy Conversion and Management, vol. 111, pp. 125-136, 2016.

[17] F. Z. Zoubiri, R. Rihani, and F. Bentahar, "Golden section algorithm to optimise the chemical pretreatment of agroindustrial waste for sugars extraction," Fuel, vol. 266, Article ID 117028, 2020.

[18] G. Gao, N. Sun, S. Dong, Y. Tao, and W. Wu, "A unified analysis for the free vibration of the sandwich piezoelectric laminated beam with general boundary conditions under the thermal environment," Shock and Vibration, vol. 2021, Article ID 1328886, 21 pages, 2021.

[19] W. Shi, Y. Liu, H. Gong, and C. Li, "Application of golden section method in unconstrained multivariate optimization problems," Journal of Northeast Normal University, vol. 35, no. 3, pp. 11-14, 2003.

[20] X. Liu, Z. Mei, and S. Zhang, "618 method, Fibonacci method, and parabola method to search for extreme points of the compound pendulum period," Physics Experimentation, vol. 30, pp. 35-38, 2010.

[21] R. Qian, "A method for optimizing smoothing coefficient using 0.618 optimization method," Systems Engineering, vol. 59-61, 1994.

[22] J. Zhang and J. Chen, "Two fast one-dimensional search methods," in Proceedings of the Seventh Academic Exchange Conference of China Operations Research Society, vol. 1, pp. 73-78, Wuhan, China, 2004.

[23] Z. Zhang, F. Jin, and Y. Tang, "An improved one-dimensional search index optimization algorithm," Journal of Huaqiao University, vol. 33, pp. 503-505, 2012.

[24] Y. Liu, "Some discussions on the golden section method," Electromechanical Technology", vol. 13-14+59, 2006.

[25] Y. Li, X. Qin, H. Cong, and M. Luo, "Research on optimum design of sections of large aspect ratio concrete independent foundation section," Industrial Architecture, vol. 48, pp. $128-132+168,2018$. 